\title{
Acute Pneumonia Today: The Worship of Antibiotics and The Neglect of Biological Laws
}

\author{
Igor Klepikov* \\ Emeritus Professor, USA
}

*Corresponding author: Igor Klepikov, Professor, Renton, Washington, USA.

To Cite This Article: Igor Klepikov. Acute Pneumonia Today: The Worship of Antibiotics and The Neglect of Biological Laws. Am J Biomed Sci \& Res. 2019 - 2(1). AJBSR.MS.ID.000567. DOI: 10.34297/AJBSR.2019.02.000567

Received: February 09, 2019 | Published: March 22, 2019

\begin{abstract}
The results of studies and clinical trials of pathogenetic approaches in the treatment of acute pneumonia (AP) were obtained in the period from 1976 to 1985 on the experience of treatment and monitoring of 994 patients. Over the past period, there has been a steady decline in the effectiveness of antibiotic therapy, an increase in the number of complications in AP with frequent development of dramatic situations, the lack of expected changes after the start of vaccination, the deficit of strategic plans to solve the problem. All these reasons only increase the relevance and timeliness of the proposed materials. The main attention is paid to biological stereotypes of dynamics of acute inflammatory processes of nonspecific etiology. Underlying the inflammatory transformation of tissues vascular reactions determine the fundamental difference between the pathogenesis and clinic AP from other lesions of this nature, as this disease is the only one in this series, which is localized in the small circle of blood circulation. The proposed principles of pathogenetic treatment are based on new ideas about the nature of AP and the unique mechanisms of its pathogenesis. The results obtained give confidence in the possibility of guaranteed prevention of complications of the disease.
\end{abstract}

Keywords: Acute Pneumonia; Pathogenesis; Schwiegk's reflex; First aid

Abbreviations: AP: Acute Pneumonia; CVB: Cervical Vago-Sympathetic Blockade; CupT: Cupping Therapy; RPG: Rheopulmonography; RR: Respiratory Rate; RV: Respiratory Volume; MV: Minute Volume of Ventilation; SA: Systolic Wave Amplitude; MBF: Minute Pulsatory Blood Flow; $\mathrm{Kv} / \mathrm{p}$ : Coefficient of Ventilation-Perfusion Ratio

\section{Introduction}

Acute pneumonia (AP) for a long period of its fame has always been considered an inflammatory disease of nonspecific etiology. Such cardinal feature of infectious diseases as contagion never appeared in descriptions of AP as this characteristic had no actual confirmation. The mental perception and importance of microbial factor in AP began to change after the discovery and use of antibiotics. Moreover, such a transformation of views on the nature of AP has a paradoxical feature: as the effectiveness of antibiotics decreases, all treatment failures are explained only by the peculiarities of the microflora. Therefore, AP as a nosological form has not changed its cardinal features, but in recent years it is increasingly represented and interpreted as an infection. This "infectious bias" in terminology and classification would be logical and justified if there were real evidence, such as contact transmission and epidemics. However, the idea of the nature of AP as an infectious disease is nothing more than a consequence of a long therapeutic fascination with antibiotics, which continue to be considered a universal remedy and panacea.

When the totality of all available information on the treatment of AP indicates that the solution to the problem does not contain real prospects, the search for a way out of this situation should begin with a radical revision of the entire strategy for the development of the disease. The first results of the work done in this direction were obtained more than 30 years ago. The initial publication of these materials took place only in Russian and remained out of the attention of the vast majority of specialists. The lack of significant advances in the treatment of patients with AP over the past period of time only adds to the importance of this area of research. The main fragments of this work are presented below and can at least give readers food for thought and shake faith in the correctness of modern ideas about AP.

The maximum volume of the journal article does not allow to give a full description of the research and its results. Therefore, this report presents only the most significant sections of the study.

\section{Material and Methods}

Studies were conducted in the Clinic of pediatric surgery at The State Institute for Advanced Training of Doctors (Novokuznetsk, USSR) in 1976-1985.During this period, the Department treated 994 children aged 4 months to 14 years with various forms of 
so-called community-acquired pneumonia and its purulentdestructive complications. Many patients from this number were selectively hospitalized in our Department in the initial period of the most aggressive forms of AP immediately at diagnosis. The reason for hospitalization of the last group of patients is that the surgical clinic was the only place of intensive care in our region with a population of about 2 million people. This group of patients was characterized by high mortality and rapid development of pleural complications. Unsatisfactory results of traditional treatment (massive doses of antibiotics, oxygen supply, methods of bronchial drainage, intravenous infusion) and a large concentration of very severe patients are forced to look for effective solutions.

The work began with a review of theoretical ideas about the nature of $\mathrm{AP}$, its causes and mechanisms of development. For this purpose, known scientific data from certain biomedical rules and laws were used. To clarify some of the nuances of the disease and obtain objective evidence of the measures taken, special studies and experiments were conducted.

\section{Experimental research}

4 series of experiments were carried out on 44 rabbits. Model of AP was created. In the last AP series of experiments (15 animals) at the time of the development of inflammation in the lung is further carried out intravenous infusion (in 6 animals into solutions for the infusions added dye-methylene blue). In this series of experiments, all animals had pneumonia with pleurisy and was later obtained a patent for the invention [1].

\section{Rentgenoanatomical studies of lung specimens}

For this investigation 56 whole lung anatomical preparations were taken during an autopsy of children who died from pneumonia. Separate contrast lung vessels (arteries or veins) with subsequent $\mathrm{x}$-ray was made on 47 specimens (in 9 cases contrasted bronchi).

\section{Comparative rheopulmonography (RPG)}

For registration and objective assessment of certain procedures of first aid when AP was used comparative RPG. Record indicators was carried out prior to and immediately after the procedure. The electrodes for recording were applied on the side of the chest wall at the level of 3 - 4ribs. Electrodes remained in the same place for the implementation of re-recording. Recording was carried out on the RPG-4 unit, "Elkar" (USSR). The age of patients these studies ranged from 3.5 to 14 years. Comparative RPG allowed to evaluate the respiratory rate (RR), respiratory volume (RV), minute volume of ventilation (MV), heart rate (HR), systolic wave amplitude (SA), minute pulsatory blood flow (MBF), coefficient of ventilationperfusion ratio $(\mathrm{Kv} / \mathrm{p})$. The results were subjected to statistical processing.

The registration of comparative RPGs was used to objectively evaluate the following procedures.

A. Cervical vago- sympathetic blockade (CVB) performed with $0.25 \%$ solution of novocaine on the side of inflammation. This type of blockade was allowed for clinical use and widespread in the Soviet Union, where the work was performed. These blockades were mainly recommended for carrying out differential diagnosis between abdominal syndrome of AP and acute appendicitis in children.

B. Cupping therapy (CupT) performed at the rate of 1 cup (50 cubic centimeters) to $4 \mathrm{~kg}$ body weight.

Comparative RPG parameters were recorded in 22 children after CVB and in 14 children after the CupT. It should be noted that cold wet wraps for a few minutes were also used as first aid to patients entering the Department. This procedure was usually performed in patients with high fever and had an optimal clinical effect. This fact requires mentioning, although, unfortunately, it was not possible to obtain objective confirmation of the positive effect in this group of patients.

\section{Scientific Basis, Additional Research and Discussion of the Results}

Among the huge number of known modern medicine inflammatory processes of nonspecific etiology only AP is the only disease that occurs and develops in the pool of the small circle of blood circulation. This fact is not in doubt and does not require further discussion. It should simply be accepted as an existing reality and as an important feature of the AP. This statement is a specific and very important characteristic of AP, since the interdependence and fundamental differences between small and large circles of blood circulation are sufficiently studied and well known. For synchronous and coordinated work of the right and left parts of the heart, equal volumes of blood are needed. Maintaining this balance is due to the preservation of the inverse proportions of blood pressure between the two circles of blood circulation and is regulated reflexively (Schwiegk's reflex).

Highly sensitive to any stimuli vessels of the small circle in the case of AP are involved in the inflammatory process, which, like any inflammation, regardless of location, is accompanied by five classic signs that reflect the severity of tissue damage. These signs of inflammation, which were described by Celsus and Galen centuries ago, are well known because they remain at the heart of our understanding of this pathology. AP is a classic inflammatory process and has all of the above symptoms-heat, pain, redness, swelling and loss of function. It is easy to note that among the presented signs of inflammation the fifth sign (loss of function) is of the greatest importance, since the specificity and severity of its clinical manifestations depend on the localization of the process.

In this regard, it should also be noted the well-known fact that $\mathrm{AP}$, as a rule, occurs without the appearance of the second classic sign of inflammation(pain), due to the lack of pain receptors in the lung tissue itself. The appearance of pain syndrome in AP usually indicates the participation of pleural sheets in the inflammatory process. However, other types of receptors, in particular baroreceptors, which were in the area of inflammation, cannot avoid the irritating effects of inflammatory tissue transformation, and this fact can significantly affect the state of blood circulation in the body. 
Continuing the return to the known scientific facts about inflammation, it is impossible not to recall that the inflammatory transformation of tissues occurs due to the vascular reaction, which is based on successive stages of changes in blood flow, blood filling, permeability of the vascular wall. In the case of AP, especially its aggressive forms with lobar lesion, the anatomical picture of these stages has long been described and is well known [2].

Important features of inflammation as a process, which should be noted in addition to the above, are the intensity and speed of the inflammatory reaction and the chain of subsequent General disorders in the patient's body. These characteristics are individual and are determined, on the one hand, by the state of the socalled reactivity of the organism, and, on the other hand, all these processes are opposed by the protective and adaptive capabilities of a particular person. The combination and counteraction of these two factors gives an infinite number of observed variants of the development of the same disease. Therefore, inflammatory changes in the tissues in the affected area can range from minor edema and infiltration to necrosis, and General changes in the body are manifested from subtle signs to the development of shock conditions.

Having remembered some fundamental data of medical science which are undoubtedly familiar to the reader, it is logical to pass to the analysis of our clinical material in the light of this information. As noted above, in our Department were concentrated patients with the most severe, aggressive current forms of pneumonia in the initial period of the disease. The dramatic state of the patients was considered as a result of severe intoxication and dehydration due to increased perspiration and high temperature. The understanding of falsity and groundlessness of the last terms came later in the course of work and additional researches. Thus, the term "intoxication" seems to be very amorphous and has no clear objective contours. It continues to be widely used in situations where there are no precise arguments to explain the severity of the patient's condition. The term "dehydration", which tries to explain the severity of the condition of patients with OP in the first hours of the disease, is generally far-fetched, since in this period of time, as a rule, there are no serious reasons for significant fluid loss, and at the heart of such a manifestation is the redistribution of circulating blood(the reasons for these shifts are listed below). However, at that time, according to the logic of these conclusions and their interpretation, each patient as a first aid at admission began to receive intravenous infusion of solutions. The harder the patient's condition was assessed, the more intensive the infusions were performed.

Powerful intravenous antibiotic therapy, as a rule, did not lead to decisive changes in the course of the disease. The process is sometimes progressed literally by the hour. Such a striking, catastrophic development of clinical and radiological picture of the disease was also an explanation, as our generation was brought up in fear of Staphylococcus. But the horror before Staphylococcus was not combined with the fact that when sowing pus from the affected area, other microorganisms were detected or the material under study was sterile. As a typical example of such development of AP in the initial period of our work can serve as the following radiographs (Figure 1 \& 2).
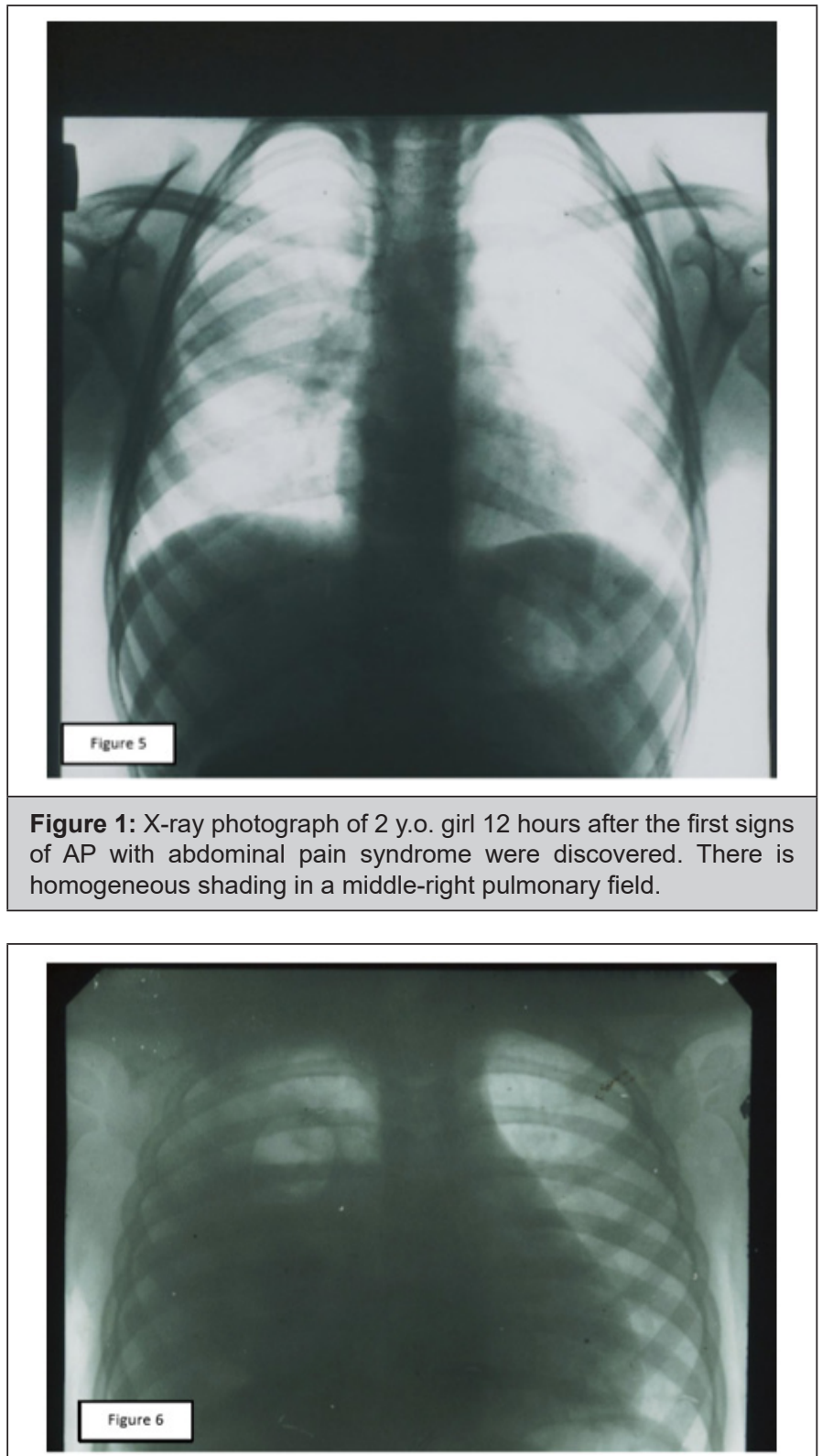

Figure 2: X-ray photograph of the same patient (see Figure 1), 36 hours after starting the inpatient care (antibiotic therapy, intravenous infusion therapy with detoxification). There is an intensive homogenous shading of almost the whole right hemithorax with mediastinum, dislocation to the left, and a cavity with a fluid level in upper pulmonary field. (Bacteriological examination of pus from the pleural cavity revealed no microflora).

Additional confirmation of the negative role of intravenous infusions in the initial period of AP, obtained during the experimental reproduction of disease models. In the last series of experiments, where the beginning of the inflammatory process was accompanied by intravenous infusions, a sample of parapneumonic pleurisy [1] was obtained stably. In addition, the principle of the discoverer of the permeability factor $V$ Menkin [3] was applied in 6 animals with additional intravenous dyes for their penetration and accumulation in inflamed tissues (Figure 3). 


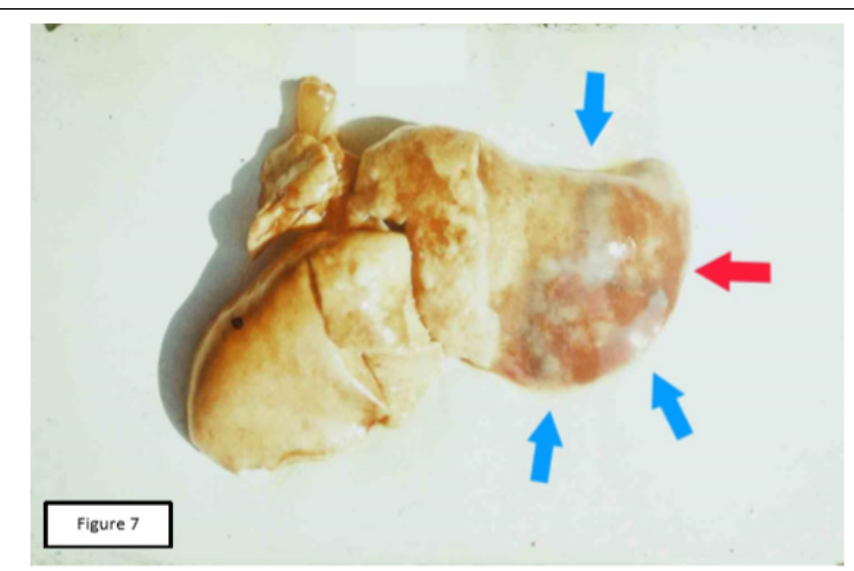

Figure 3: Macro-preparation of the lung, experiment, series 4b. Massive focus of the inflammation in a pulmonary surface (red arrow), surrounded by the additional sections of infiltration with blue shading (blue arrows).

In parallel with clinical observations and experimental results, $\mathrm{x}$-ray anatomical studies of lung specimens with varying degrees of inflammatory changes provided additional information. As a result of the occurrence of edema and increased tissue infiltration in the focus of inflammation, a gradual compression of the vessels occurs. At the same time, more pliable venous vessels suffer in the first place, which creates an obstacle to the outflow of blood with preserved arterial provision. This variant of anatomical transformation of blood vessels in the area of progressive inflammation forms a kind of vascular trap, in the presence of which an additional increase in blood flow in intravenous infusion is a catalyst for further tissue infiltration to the end of blood flow. In the most severe situations, you can find additional signs of compensatory restructuring of the vascular bed of the non-inflammatory parts of the lung as a reflection of the overload of the small circle of blood circulation (Figure 4).

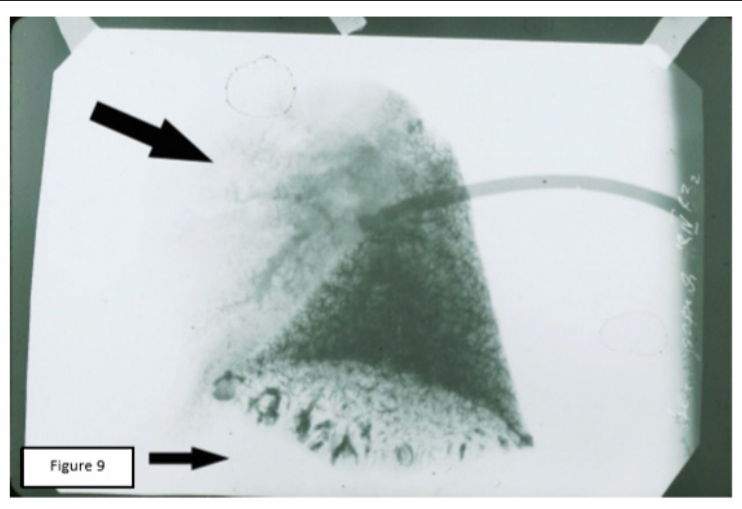

Figure 4: Venogram of lung's preparation with a massive focus of the inflammation in the upper portion (histology which followed, did not show the features of the necrosis in inflammation's focus). Sharp depletion of the venous picture in the upper portion of the lung. Lower portion shows relatively large vascular formations of a halfring shape with a well-contrasted venous vessels in the background.

Direct and indirect evidence of the risk of intravenous infusions during the progression of edema and infiltration of pulmonary tissue dictated the need to abandon them as a first aid in AP. Simultaneous change of ideas about the nature of pathological processes in the
AP required a radical revision of the complex of medical care and the choice of adequate pathogenetic methods. In the latter case, the choice was made in favor of methods that are already periodically used in this category of patients and which created an undoubted subjective impression of their effectiveness. However, we were not satisfied with the subjective criterion of assessing the impact of these procedures and therefore to obtain objective data on the impact of certain techniques on the dynamics of certain indicators used available at the time opportunities.

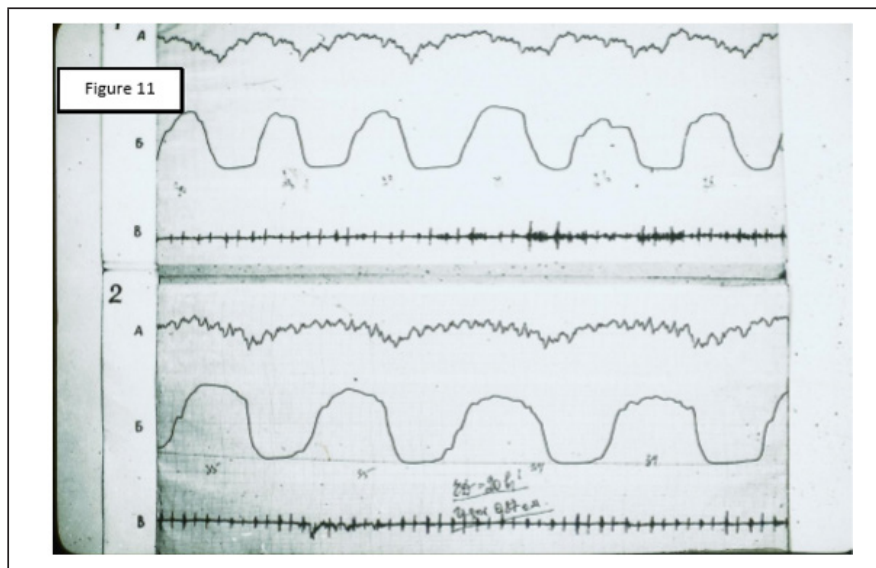

Figure 5: Rheogram of ventilation of the patient 8 years, AP - 7-10 segments on the right lung. 1: The source RPG; 2 : RPG after cupping therapy; A: Differential RPGs; Б: Main RPG; B: Phonocardiogram.

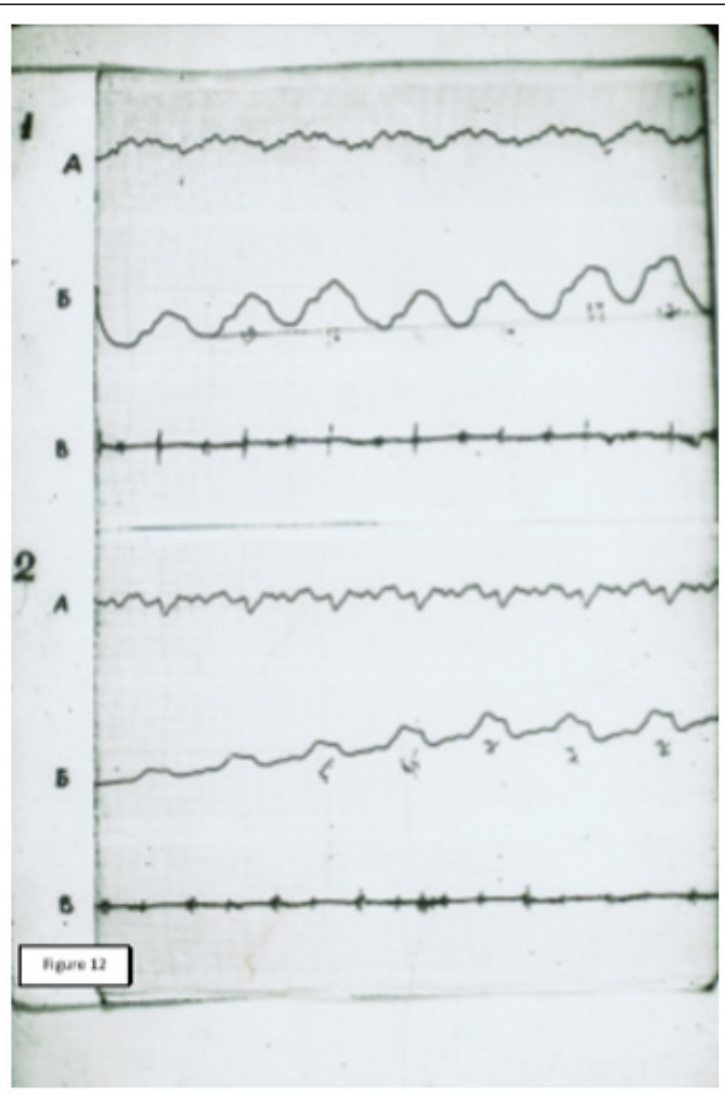

Figure 6: Rheogram of pulsatory blood flow of the same patient. 1: The source RPG; 2: RPG after cupping therapy; A: Differential RPGs; Б: Main RPG; B: Phonocardiogram. 
The result of these intentions were the records of comparative RPGs, which can serve as objective evidence of the pathogenic effectiveness of CVB and CupT. The analysis of the obtained records allowed us to note that, despite the fundamental differences in the place of application and technique of these two procedures, their final results were almost the same. The values of RR and MV after the procedure decreased significantly, although RV remained unchanged. Performance SA, MBF has changed markedly, HR decreased to a lesser extent. In General, the data obtained showed a significant decrease in hyperventilation and improved perfusion. Baseline values of RPG indicated a marked predominance of ventilation over blood flow. After treatment, the ratio between these parameters $(\mathrm{Kv} / \mathrm{p})$ was equalized. This point was accompanied by a significant improvement in the condition and well-being of patients. A visual representation of change records RPG under the influence of CupT gives a comparative picture of these curves, which were recorded from one of our patients (Figures 5 \& 6).

Thus, this description presents only a part of the whole study, the main result of which was the creation of a new doctrine of the AP. This stage is key and relates to the study of a number of interrelated and consistent mechanisms of pathological changes at different levels that occur with the onset of inflammation in the pulmonary tissue and determine the dynamics of AP. In this regard, on the basis of the above material, we can briefly present the developing pathological transformations, their interdependence and dynamics in the field of inflammation, and in the body as a whole. At the same time, both focal and General processes have a typical scenario of development, but the severity and demonstrativeness of the manifestations of the disease remain an individual feature of each patient.

The inflammatory transformation in the lesion is caused by a standard vascular reaction with a radical restructuring of the blood flow and an increase in vascular permeability. With the accelerated development of these processes, a rapid increase in the volume of inflamed tissues leads to the development of the so-called vascular trap, which further disrupts the blood flow, until he stops. During this period of the disease intravenous infusion increases blood flow in the area of inflammation and only activates the pathological process. Excess tissue fluid in such situations appears faster and accumulates in the pleural cavities, and the frequency of parapneumonic effusions among patients receiving infusion is significantly higher than among patients who have avoided dropper.

Inflammatory changes in the vessels of the small circle of blood circulation create a focus of irritation, which plays the role of the reflexogenic zone. Generalized vascular spasm of the small circle, on the one hand, is a natural attempt of the body to reduce blood flow to the focus of inflammation and reduce the degree of pathological changes. However, on the other hand, with increasing pressure and obstruction of blood flow in the vessels of the small circle, the Schwiegk's reflex is triggered, which as an adaptive reaction is aimed at reducing pressure in the large circle and reducing blood flow to the pulmonary vessels.

The resulting restructuring of the systemic blood flow depends on the rate of its changes. The faster the blood circulation conditions change, the less chances and opportunities for the body to adapt to them and neutralize their consequences. Therefore, in the most severe cases, we can observe a typical picture of shock. This variant of shock is distinguished by its origin from other known species. The main and leading cause of shock if AP is a reflex action of the tumor in the lung into systemic circulation. Timely elimination of the reflex mechanism of the disease by SVB or CupT, even in the presence of bacteremia, gives a visible effect in a few minutes. This unique variant of shock was singled out separately and described earlier as "pulmonary shock" [4-6], which, unlike other types of this pathology, requires fundamentally different approaches to treatment.

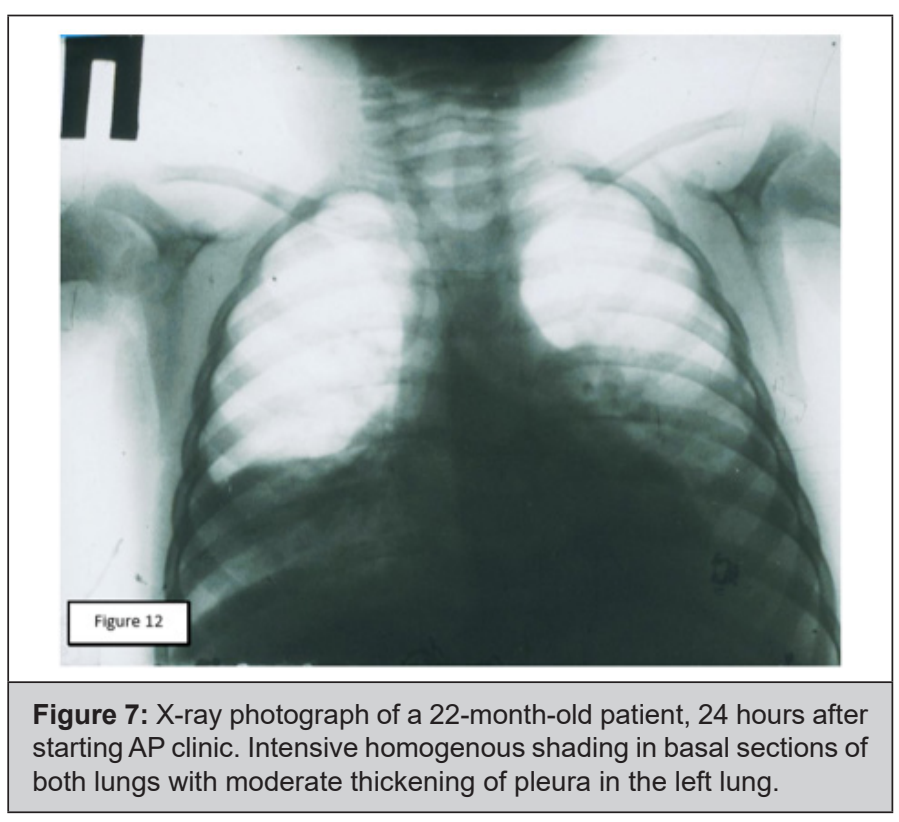

The information presented and its interpretation were incorporated as an integral part of the new AP doctrine and served as a justification for the subsequent revision of the treatment principles for this group of patients. New pathogenetic approaches in treatment were used in 101 patients with signs of infiltrative changes in the lungs. Another 102 patients treated according to the revised principles had a pleural reaction at the time of admission. The final results allowed to speak about the possibility of guaranteed prevention of complications of AP. The use of pathogenetic approaches to treatment in the initial stage of the so-called "toxic" forms of AP ( CVB , CupT, cold wraps of patients, intensive restoration of bronchial patency, rejection of intravenous infusions , intramuscular or intravenous administration of antibiotics with a minimum volume of solution) showed that in comparison with the same group who received the previous first aid complex, the number of pleuro-pulmonary complications is significantly reduced (in the group of patients who did not have a pleural response at admission ,101 patients, $\mathrm{T}=8.65 ; \mathrm{P}<0.001$ ), the length of stay in the hospital decreased by three times, respectively, material and financial costs of treatment were significantly reduced, there were no deaths. A detailed description of the whole complex of works was first published in Russian [7], and last year-in English [8]. One of our observations can serve as an illustration of new approaches in the treatment of patients with AP (Figure 7 \& 8). 


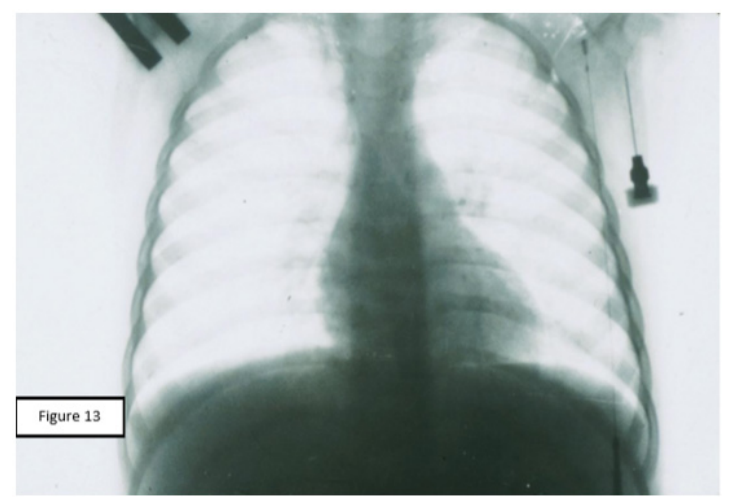

Figure 8: X-ray photograph of the same patient (see Figure 7) five days following the clinical treatment (CVB, antibiotic therapy, bronchus-draining therapy, including lavages through microtracheostoma). Full recovery of airiness in both lungs.

There is almost no doubt that the majority of readers after reading the above materials are in a state of bewilderment and uncertainty, since the usual designation of microbial pathogens and means of their suppression is not reflected in this text. To explain this situation, it should be absolutely clear that the principles and use of antibiotic therapy have not changed and have not disappeared. There has only been a change in emphasis and priorities in the choice of methods of assistance. The choice of antibiotics both in that period and today occurs empirically, and the causative agent of AP in the vast majority of cured patients anywhere in the world remains unidentified. In the light of recent facts, the standard list of the most likely AP agents, which is usually contained in manuals and major analytical articles, has a more declarative and frightening than practical meaning. The reader can pay attention to two examples that were given above. At the first observation, the sowing of pus from the pleural cavity (after the start of antibacterial therapy) did not reveal microflora. In the second observation, no bacteriological examination was carried out at all. And what was the significance of these facts for the choice of medicines and treatment results?

The topic of the aetiology of the AP can be the subject of a separate report, taking into account the experience of reproducing the model of this process and its complications in the experiment. Here it should be noted that the microbe is one of the factors that provoke inflammation in the lung tissue, but not the only reason. The fact that " antibiotics alone " outpatient therapy still helps many patients with AP should be regarded as a temporary success of pharmacists in creating new generations of antibiotics in response to the emergence of more resistant bacteria. Preservation of "antibiotics alone" adequacy in some patients with AP suggests that their body is enough to suppress the pathogen, so that in the future he coped with the disease on their own. However, the problem becomes apparent when initial treatment fails to meet expectations. The progression of the disease in the background and, despite treatment, is irrefutable proof of the discrepancy of these efforts in the pathogenesis of the process. Such observations, which probably were in the past for each practitioner, are the best proof that antibiotics are not an absolute means of treating AP and do not have a direct effect on the ongoing process of inflammation. It is these situations that make us remember the already known biological rules and laws, the action of which exists and continues regardless of our perception. At the same time, it is clear that our professional assessment of the situation and the direction of our follow-up significantly depend on the level of our perception of such real circumstances. Ignoring the attention to the known biological rules and laws, we only narrow our understanding of the nature of the disease and limit the search for targeted pathogenetic measures of influence on the inflammatory process.

\section{Conclusion}

The development of the focus of AP occurs in accordance with biological stereotypes of the dynamics of acute inflammatory processes of nonspecific etiology. Underlying the inflammatory transformation of tissues vascular reactions determine the fundamental difference between the pathogenesis and clinic AP from other lesions of this nature, as this disease is the only one in this series, which is localized in the small circle of blood circulation. The proposed principles of pathogenetic treatment are based on new ideas about the nature of AP and the unique mechanisms of its pathogenesis. The results obtained give confidence in the possibility of guaranteed prevention of complications of the disease.

\section{References}

1. Klepikov I, Rikov V (1990) A method for obtaining a model of parapneumonic pleurisy. Author's certificate for invention. SU, No 1631574, A1, 1 .

2. Lobar pneumonia: https://en.wikipedia.org/wiki/Lobar_pneumonia.

3. Menkin Valy (1940) Dynamics of inflammation. Macmillan.

4. Klepikov I, Yudin J (1986) Shock in cases of acute bronchogenic pneumonia. A collection of articles Clinical and anatomical aspects of disseminated intravascular blood coagulation and shock. Leningrad, Leningrad Institute for Advanced Training of Doctors p. 53-55.

5. Klepikov I et al. (1986) Characteristic of circulatory and metabolic lesion in cases of acute bronchogenic pneumonia in children. A collection of articles Clinical and anatomical aspects of disseminated intravascular blood coagulation and shock. Leningrad, Leningrad Institute for Advanced Training of Doctors p. 68-69.

6. Klepikov I (1988) Anti-shocked therapy in cases of acute bronchogenic pneumonia. Medical science for practice (Theses of Scientific and Practical Congress), Novokuznetsk p. 73.

7. Klepikov I (1989) Acute pneumonia and its purulent and destructive complications in children in the midst of a major industrial centre of Western Siberia. Dissertation for the degree of Doctor of Medical Science. Leningrad.

8. Igor Klepikov (2017) Acute pneumonia: a new look at the old problem. Lambert Academic Publishing. ISBN (978-3- 330-35250-6). 